\title{
An adaptive data distribution system for mobile environments
}

\author{
Sascha Kümmel, Alexander Schill, Karsten Schumann, Thomas \\ Ziegert
}

Dresden University of Technology, Department of Computer Science, Institute for Operating Systems, Databases, and Computer Networks D-01062 Dresden, Germany, Tel.: +49 3514575 457, Fax.: +49 351 4575 251, \{ kuemmel, ziegert\}@ibdr.inf.tu-dresden.de

\begin{abstract}
Common transport systems lack the appropriate mechanisms to deal with the problems of mobile computing systems (e.g. temporary inaccessibility, transient network addresses of mobile hosts, along with varying quality of service parameters of physical network connections). Therefore, a need for new adaptive data distribution mechanisms.

This paper discusses specific features of GISMO's (Generic Infrastructure Support for Mobile Objects) infrastructure; in particular, a mobile queuing service - which distributes data in an adaptive manner. In the remainder of this paper we introduce and motivate the need for adaptive data distribution mechanisms, describe our concept, as well as some implementation details and an overview of our first experiences with a prototype implementation.
\end{abstract}

\section{Keywords}

adaptation, data distribution, disconnected operation, mobile computing, mobile transport system, queuing systems

\section{INTRODUCTION}

During the last few years the fields of mobile computing and mobile communications has make considerable progress which has lead to a strong trend towards integrating mobile hosts within existing data networks. Mobile hosts appear at different locations at different times. During moves and connections over wireless links we have a situation where frequent disconnections occur. Furthermore, the bandwidth of common wireless communication infrastructures is limited (Mello, 93), (Davies, 94). Other problems are: resource heterogeneity, security and the management of location dependent data. 
One way to overcome these problems is to implement new applications from scratch. Due to the similarity of a significant number of applications concerning the above mentioned issues, we outline the importance of a support platform for mobile applications giving generic assistance in a reusable manner. Considering this, we are currently developing and implementing a Generic Infrastructure Support for Mobile Objects (the GISMO-Project). The basic design of our support platform is presented by (Schill, 95). This paper discusses specific features of this infrastructure, in particular a mobile queuing service.

\section{MOTIVATION}

Common transport systems lack the appropriate mechanisms to deal with temporary inaccessibility, transient network addresses of mobile hosts, along with varying quality of service parameters of physical network connections.

There are some considerable efforts to solve the problem of disconnected operations and temporary inaccessibility ((Huston, 93), (Huston, 95), (Kistler, 92), (Satyanarayanan, 93) and (Satyanarayanan, 94)). These solutions mainly focus on file systems, based on caching, operation logging and reintegration. A recoverable queuing service for distributed transaction processing as a solution for reliable operation handling in case of inaccessibility is described in (Dietzen, 92) and (Transarc, 94). But they did not deal with service mobility and QoS adaptation. (Balakrishnan, 95) shows a way for the improvement of TCP/IP performance over wireless networks and (Bakre, 95) enhances the RPC-mechanism with mobility awareness.

While working on GISMO, the need of a new adaptive data distribution mechanism arose. This mechanism has to cope with postponed data transfer by intermediate persistent storing, „mobile addressing“ supported by special locating mechanisms, detection of QoS parameters and appropriate adaptation during the transfer.

QoS adaptation can be achieved by varying the data packet size depending on the average error rate and by data conversion or compression. The parallel transfer of data packets by multiple threads in case of long delay connections obviously decreases the transmission time, see (Kümmel, 95).

\section{THE CONCEPT}

In this paper we focus on the data transport layer and the disconnected operation handling within the GISMO-architecture (for a detailed description see (Schill, 95)). For better understanding we use an e-mail system as an application scenario below (details can be found in (Schill, 96)).

We present a transport system according to the queuing principle, the queuing service (QS). The QS is designed to support transfer of data units in any size, so called databodies. It is possible to describe dependencies between databodies. This allows the transfer of complex data structures with explicit access to each element by the QS. The explicit access enables data format detection and conversion in advance (before the transmission). A suitable example therefore is a multimedia e-mail, composed of text documents, audio and/or video and other attachments. By building an e-mail using single databodies for each type of information, the 
user is able to select only the databodies he really wants in case of a low-bandwidth connection.

It is possible to assign the QS to reduce the size of a bodypart in advance. This allows the reduction of costs and time during a transmission over a low bandwidth link,. The reduction may cause a loss of quality, so we believe that user interaction during the selection of the rather subjective compression parameters is very desireable. For an automatic adaptation 'on the fly' we suggest another solution. The data source should deliver further information about the maximum possible compression, or on the other hand which parts of the information are vital to be transmitted in any case. The QS data structures enable the transfer of additional data, so this kind of operaion is supported.

For the evaluation of QoS parameters, the assessment and conversion respectively compression of different media types, external services within the GISMO System will be deployed. The QS is only aware of the fact, that the need for data conversion and QoSdetection exists.

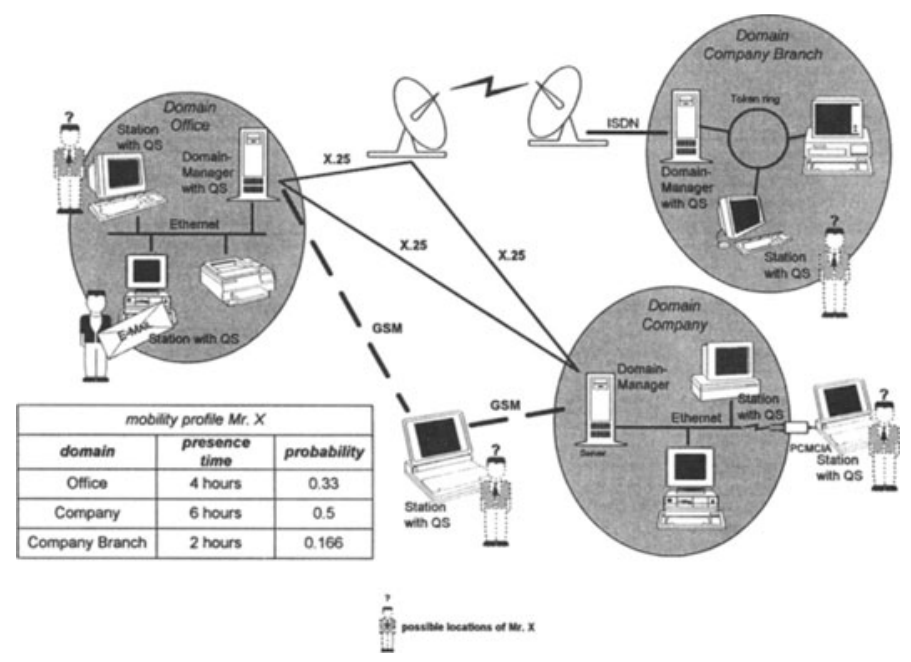

Figure 1 a sample environment

Figure 1 shows a sample environment. Queuing systems run on every station within the distributed environment. There is no central queuing server, due to the fact that redundancy and optimum routing are supported. Target addressing is not only limited to network addresses, it is also possible to use a list of target descriptions in order to meet the mobile aspects. In the example e-mail application a description is derived from a user identifier and an application identifier (we use in our prototype universal unique identifiers). Target locating is achieved by an external service - the Application Data Mobilizer and Manager (ADMM; for further explanation see (Schill, 95)).

The following short description of one distribution cycle is based on figure 2. The source application enqueues the databody together with a destination description (1). The ADMM 
locates the QS which is currently assigned to the target application and the respective user (for mechanisms for distributed location of mobile objects see (Dasgupta, 94)) and assembles a routing path (2). In case a user is not connected and hence it is impossible to locate him, the ADMM supports mobility profiles which include possible locations of the users. Users usually relocate to a lazy changing set of locations, see also figure 1 . Therefore the QS supports multicast data transfer to all probable destinations. The distribution is controlled by the routing path obtained from the ADMM and data is sent hop by hop. There can be several multicast distribution hops in the path for optimum exploitation of available bandwidth. Upon reception of a databody at the QS it is possible to contact the ADMM (3). So adaptation to changing network topology and very high target mobility are supported. We call it „,adaptive source routing“.

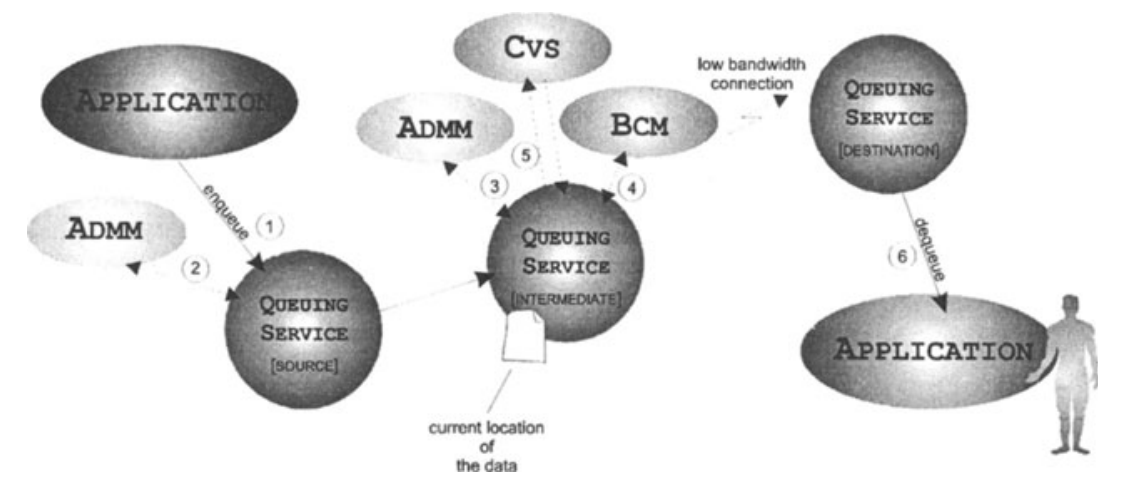

Figure 2 the queuing service

On every intermediate QS the physical connection to the next QS is evaluated by the bandwidth and cost manager (BCM) (4). A conversion service (CVS) converts the databody into another format with a significant reduction of its size in case of a low bandwidth connection or high costs and if the databody is indicated as convertible (5). The modified databody is dequeued by the destination application (6).

The transfer between two QS is covered by a transaction-like protocol and optimized accordingly to the currently available QoS parameters. All received data is stored persistent to ensure reliability and recovery in case of a system crash or network breakage. To support multicast distribution and to protect the system from data overflow we implemented lifetime control and garbage collection mechanisms. Each databody has a maximum lifetime. After expiration, the databody and all its copies will be removed from the whole distributed system. If a databody is dequeued by an application, a garbage collection process is started which will remove all copies within the system and inform the source application the successful operation has completed.

In order to consider security requirements, security tags (to support various security techniques) will also be transferred with every databody. All distribution steps could be verified based on these tags using an external security component. 


\section{THE IMPLEMENTATION}

The basic architecture of the queuing service is shown in figure 3. It is made of five core components and multiple in/out data queues to support priority queuing (we distinguish between hierarchy and priority rather than (Athan, 93)). The QS acts as a single process with multiple threads. It uses three external services to obtain information about addressing, current QoS parameters and data conversion in advance of transmission.

The distribution control component monitors the data distribution within the mobile environment. All data transfer actions will be logged. Therefore, the component is informed by the four other components in case of data enqueuing or dequeuing and data transfer to or from a remote QS. The distribution control component itself communicates via RPC with other distribution control components running on other QS elsewhere. The logged transfer actions allow tracing the data distribution within the system. This accomplishes garbage collection, lifetime control and back reporting of distribution results.

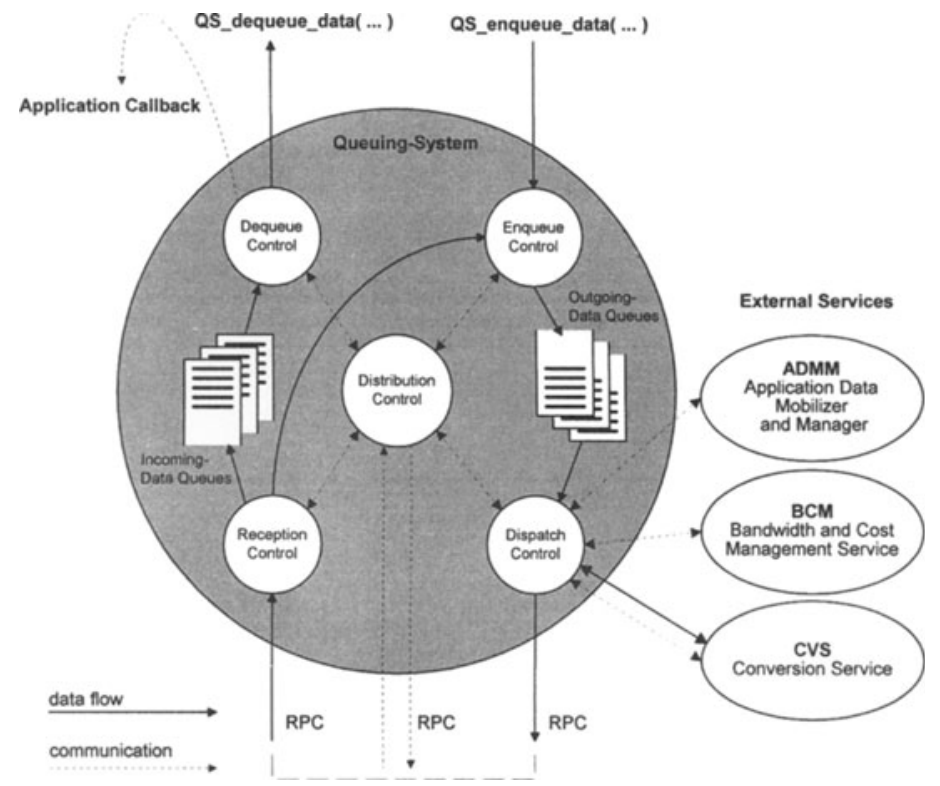

Figure 3 The basic architecture of the Queuing Service

Applications, which want to use the QS have to implement a callback procedure, so the QS can inform them about data arrival or the completion of a dequeuing operation at a remote site. Assuming a long time between the enqueue and dequeue operations there is a high likelihood that the sending application has already gone. So what will happen with the back reported message about data arrival? Applications can specify a file, so the system can log any reports about successful arrivals or failures during delivery (timeouts). 


\section{EXPERIENCES AND FUTURE WORK}

We have implemented a first prototype queuing system based on the Windows NT/95 operating systems and Microsoft RPC. The sources will be ported to UNIX platforms on top of OSF/DCE soon. So the system can run on mobile clients based on Windows95 while the servers are running on more powerful Unix workstations. We successfully built a mobile aware multimedia X.400 e-mail system on top of our prototype (see (Schill, 96)).

Currently we are able to present the first results of performance measurements with the system prototype. Figure 4 shows the average transfer times between two QS running on a PC Pentium $100 \mathrm{MHz}, 32 \mathrm{MB}$ memory each, connected by a HP 100 VG AnyLAN. The transfer time is measured between the moment an application has completed it's enqueuing operation on one QS until an other application completes its dequeuing operation on the other. The application will be informed by a callback function called by the QS if addressed data is available.



Figure 4 throughput measurement

We currently working on an enhanced transfer protocol. The protocol performs optimized asynchronous multithreaded packet transport and continuous recovery abilities for aborted transfers in case of network disconnection, disruption or station switch off. There are some further enhancements to do. Considering the worst case of a low bandwidth and cost intensive connection to a remote site and assuming a few messages in the queue waiting for delivery, we must decide, which message will be delivered first. Of course the message headers own a higher priority than other databodies within a container, but sending all headers first involves a combination of multiple operations, if an error occurs lots of things have to set up again, so it may be better to send some containers in one trial.

Future work will focus on packet transport mechanisms without using RPC respective TCP/IP to maximize throughput for low-bandwidth connections found in wireless networks, because of the known TCP problems with large and also very short delay times due to the window mechanism (for details see (Balakrishnan, 95), (Thekkath, 93) and (Kay, 93)). 
We also plan to implement mobile database access and mobile-RPC (Winkler, 95) facilities on top of our prototype. An overall transaction-management and enhanced security facilities within the system are also under development.

\section{Acknowledgments}

We would like to thank all involved colleagues and students for their significant efforts in building the implementation of the described prototype. Moreover, we would like to thank Digital Equipment GmbH (EARC Karlsruhe for sponsoring and for supplying the X.400Infrastructure).

\section{REFERENCES}

Athan, A. and Duchamp, D. (1993) Agent-Mediated Message Passing for Constrained Environments, in Proceedings of the USENLX Mobile and Location-Independent Computing Workshop, Cambridge MA, 103-7

Bakre, A. and Badrinath, B.R. (1995) M-RPC: A Remote Procedure Call Service for Mobile Clients, in Proceedings of the $1^{\text {st }}$ ACM Mobicom Conference, 2-11

Balakrishnan, H., Amir, E. and Katz, R.H. (1995) Improving TCP/IP Performance over Wireless Networks, in Proceedings of the $1^{\text {st }}$ ACM Mobicom Conference, 124-31

Dasgupta, P. (1994) Resource Location in Very Large Networks, in Proceedings of the IEEE Computer Society First International Workshop on Services in Distributed and Networked Environments (SDNE'94), 156-63

Davies, N., Pink, S. and Blair, G. S. (1994) Services to Support Distributed Applications in a Mobile Environment, in Proceedings of the IEEE Computer Society First International Workshop on Services in Distributed and Networked Environments (SDNE '94), 84-9

Dietzen S. (1992) Distributed Transaction Processing with Encina and the OSF DCE, Transarc Corporation

Huston L.B. and Honeyman P. (1993) Disconnected Operation for AFS, in Technical Report No. CITI 93-3, University of Michigan

Huston L.B. and Honeyman P. (1995) Partially Connected Operation, in Technical Report No. CITI 95-5, University of Michigan

Kay, J. and Pasquale, J. (1993) The Importance of Non-Data Touching Processing Over-heads in TCP/IP, in Computer Communications Review

Kümmel, S. and Schill, A. (1995) Leistungsanalyse und Vergleich von RPC-Systemen für heterogene Workstation-Netze, in PIK - Praxis der Informationsverarbeitung und Kommunikation, Issue No. 3, 148-53

Mello, J. and Wayner, P. (1993) Wireless Mobile Telecommunications, Byte, Februar 1993, 147-54

Satyanarayanan M. et. Al. (1993) Experience with Disconnected Operation in a Mobile Computing Environment, in Technical Report No. CMU-CS-93-168, Carnegie Mellon University

Satyanarayanan M. and Noble D.B. (1994) Coda, An Empirical Study of a Highly Available File System, in Technical Report No. CMU-CS-94-120, Carnegie Mellon University 
Kistler, J. J. and Satyanarayanan M. (1992) Disconnected Operation in the Coda File System, in ACM Transactions on Computer Systems, No. 10

Schill, A. and Kümmel, S. (1995) Design and Implementation of a Support Platform for Distributed Mobile Computing in Mobile Computing Special Issue of Distributed Systems Engineering, 128-41

Schill, A., Kümmel, S. and Ziegert, T. (1996) Mobility aware Multimedia X.400 email: A Sample Application Based on a Support Platform for Distributed Mobile Computing, in Proceedings of the IMC '96 Workshop for Information Visualization \& Mobile Computing

Transarc Corporation (1994) Encina RQS Programmer's Guide and Reference

Thekkath, C.A. (1993) Limits to Low-Latency Communication on High Speed Networks in ACM Transactions on Computer Systems; Vol.11, No.2

Winkler, M. and Kümmel, S. (1995) Mobile RPC - Eine Erweiterung des DCE Remote Procedure Call, in Proceedings of the GUUG-Jahrestagung 1995, 116-22

\section{BIOGRAPHY}

Prof. Dr. Alexander Schill is chair of the institute of Operating Systems, Data Bases and Computer Networks at Dresden University of Technology. He received his Ph.D. in Computer Science in 1989 at Karlsruhe University. In 1990/91 he worked at IBM T.J. Watsom Research Center, Yorktown Heights. His main research areas are distributed systems, high performance networking and mobile computing.

Sascha Kümmel received his diploma in computer science in 1994 from the Dresden University of Technology. He's currently working as research assistant at the institute of Computer Networks at Dresden University of Technology. His main research areas are mobile computing and high performance networking. His work is supported by Digital Equipment Corporation.

Karsten Schumann is a student of Computer Science at Dresden University of Technology since 1991. Karsten is student member of the GISMO Group. He is interested in Mobile Computing and Distributed Systems.

Thomas Ziegert received his diploma in computer science in 1995 from the Dresden University of Technology. He is currently working towards his Ph.D. at the Dresden University of Technology with a special interest in efficient location mechanisms in mobile environments. His work is supported by the German Science Foundation (DFG). Thomas is a member of the GISMO Group. 